\title{
THE OESOPHAGO-GASTRIC SPHINCTER AFTER CARDIOMYOTOMY
}

\author{
BY \\ MICHAEL ATKINSON \\ From the Department of Medicine, University of Leeds
}

(RECEIVED FOR PUBliCATION FEBRUARY 2, 1959)

Surgical operations performed for the relief of achalasia of the cardia are liable to be followed by gastro-oesophageal reflux. Although this complication occurred much more frequently after earlier procedures such as cardioplasty or oesophago-gastric anastomosis than after Heller's cardiomyotomy (Barrett and Franklin, 1949 ; Ripley, Olsen, and Kirklin, 1952 ; Brewer, Barnes, and Redo, 1956), it is nevertheless encountered in a minority of patients after the latter operation. Estimates of the incidence of gastro-oesophageal reflux after cardiomyotomy vary considerably. Hawthorne, Frobese, and Nemir (1956) found this to be present in four of 35 patients who had undergone cardiomyotomy, and Acheson and Hadley (1958) reported heartburn in 13 of 22 patients after this operation. Gertz (1952), however, did not encounter reflux in 11 patients subjected to cardiomyotomy, and Gammie, Jennings, and Richardson (1958) state that "cardiomyotomy by itself does not lead to incompetence of the cardia or to gastrooesophageal reflux."

It is now well established that the normal oesophago-gastric sphincter can be demonstrated by manometric methods as a localized zone at the oesophago-gastric junction in which intraluminal pressure is raised (Fyke, Code, and Schlegel, 1956; Botha, Astley, and Carré, 1957 ; Atkinson, Edwards, Honour, and Rowlands, 1957a).

In this study a similar manometric technique has been used to assess the functional state of the oesophago-gastric sphincter after cardiomyotomy. The purpose was to determine whether the development of gastro-oesophageal reflux after this operation depended upon the extent of the disruption of the sphincter. By this means it was hoped to provide additional information about the importance of this sphincter in maintaining the competence of the cardia.

\section{Patients Studied}

Eighteen patients who had undergone Heller's cardiomyotomy, one month to nine years previously, were studied (Table I). The operations had been carried out at several centres by many different surgeons, but in each a thoracic approach was used. The patients were specifically selected to include a large number with symptoms of gastro-oesophageal reflux and therefore no conclusions about the incidence of this complication can be drawn from the figures given. Any patient who suffered from hiatus hernia was excluded from the study. In two additional patients the pressure-recording tubes could not be passed beyond the lower oesophagus and these patients are therefore not included in the series.

Twenty-three subjects of age and sex distribution comparable with that of the patients after cardiomyotomy were studied to provide measurements from the normal oesophago-gastric junction. These consisted of students, staff, and patients without abnormality of the alimentary tract or diaphragm.

\section{METHOD}

Clinical Assessment.-Clinical criteria were used to define gastro-oesophageal reflux. The presence of pain or discomfort in the chest or throat brought on or aggravated by bending forwards or lying flat and acid-tasting fluid rising into the mouth were taken as evidence of reflux. On this basis five of the 18 patients studied were considered to suffer from reflux and each stated quite definitely that the symptoms began after the operation. None of the five patients had much radiologically demonstrable residue in the oesophagus after the operation, and hence the possibility that these were suffering from reflux from the oesophagus rather than from the stomach seemed remote.

Three patients had slight dysphagia persisting after the operation, but in each this was intermittent and seldom resulted in the return of food. 
TABLE I

FINDINGS IN CONTROLS AND PATIENTS

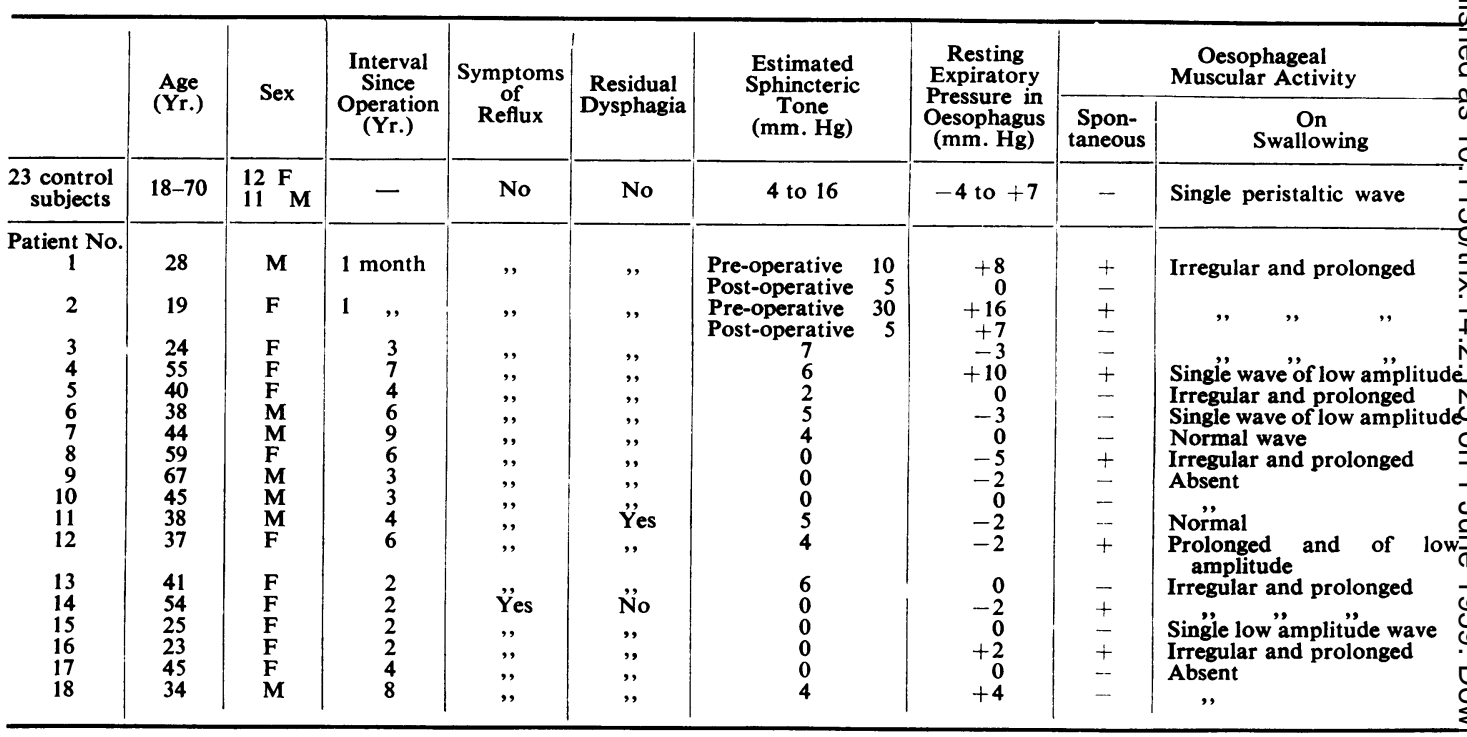

Manometric Technique.-The function of the oesophago-gastric sphincter and the pattern of muscular activity in the body of the oesophagus were assessed by recording intraluminal pressure from the stomach, oesophago-gastric junction, and oesophagus.

Pressures were measured through open-ended polyethylene tubes filled with water and connected to capacitance manometers outside the body. The theoretical volume change in the manometer was $0.16 \mathrm{c} . \mathrm{mm}$. per $100 \mathrm{~mm}$. $\mathrm{Hg}$ change in pressure. Pressures were recorded simultaneously through two such polyethylene tubes fastened together with their tips $5 \mathrm{~cm}$. apart.

All measurements were made with the patient fasting and lying on his right side to avoid any increase in pressure in the lower oesophagus caused by the weight of the mediastinal viscera. The recording tubes were passed through the nose until their tips both lay below the diaphragm. They were then withdrawn in $1 \mathrm{~cm}$. stages taking a 15-sec. record of intraluminal pressure at each level while the patient was breathing quietly. Pressures were never recorded while the tubes were being moved. When the distal tip lay at least $5 \mathrm{~cm}$. above the level of the diaphragm, as assessed by the type of respiratory deflection (Atkinson and others, 1957a), the tubes were replaced below the diaphragm and the procedure repeated at least twice. As two tubes were being used six records of pressure from stomach to oesophagus were obtained. Finally records of the pressure changes during "dry" swallows and swallows of small amounts of cold water were obtained at the oesophago-gastric junction and at various levels in the oesophagus.

\section{RESULTS}

Normal Subjects.- When the tip of the tube $\stackrel{\mathbb{2}}{\Rightarrow}$ was withdrawn in $1 \mathrm{~cm}$. stages from the stomach into the oesophagus a zone of raised intraluminal pressure $1-4 \mathrm{~cm}$. in length was seen at the oesophago-gastric junction (Fig. 1). This rise in pressure is brought about by the tonic contraction $\vec{\partial}$ of the oesophago-gastric sphincter compressing $\underset{\widetilde{x}}{\stackrel{\partial}{\hat{N}}}$ the pressure recording device. On swallowing, $;$ the pressure in this zone fell to the general intra- 3 . oesophageal level as the sphincter relaxed during the swallowing reflex (Fig. 2).

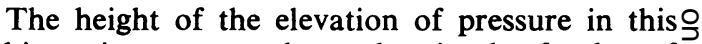
sphincteric segment above that in the fundus of $D$ the stomach was used as an estimate of the state을. of contraction or tone of the oesophago-gastric $\bar{N}$ sphincter. The values were calculated using . pressures measured during the pause at the end 0 of expiration. Measurements were made during $N$ withdrawal of a recording tip from stomach to oesophagus six times in each individual, and, 0 although pressures in the stomach and in the $\frac{}{\infty}$ oesophagus corresponded closely in different $\stackrel{\oplus}{+}$ withdrawals, a greater variation in pressure in the 0 sphincteric segment was noted. The maximal values for intraluminal pressure in the sphincteric $\stackrel{\Phi}{\Phi}$ segment were used in calculating the elevation of $\overrightarrow{\mathbb{Q}}$ pressure, which was taken as a measure of the $\overline{0}$ functional state or tone of the oesophago-gastric sphincter. The values obtained from 23 normal 


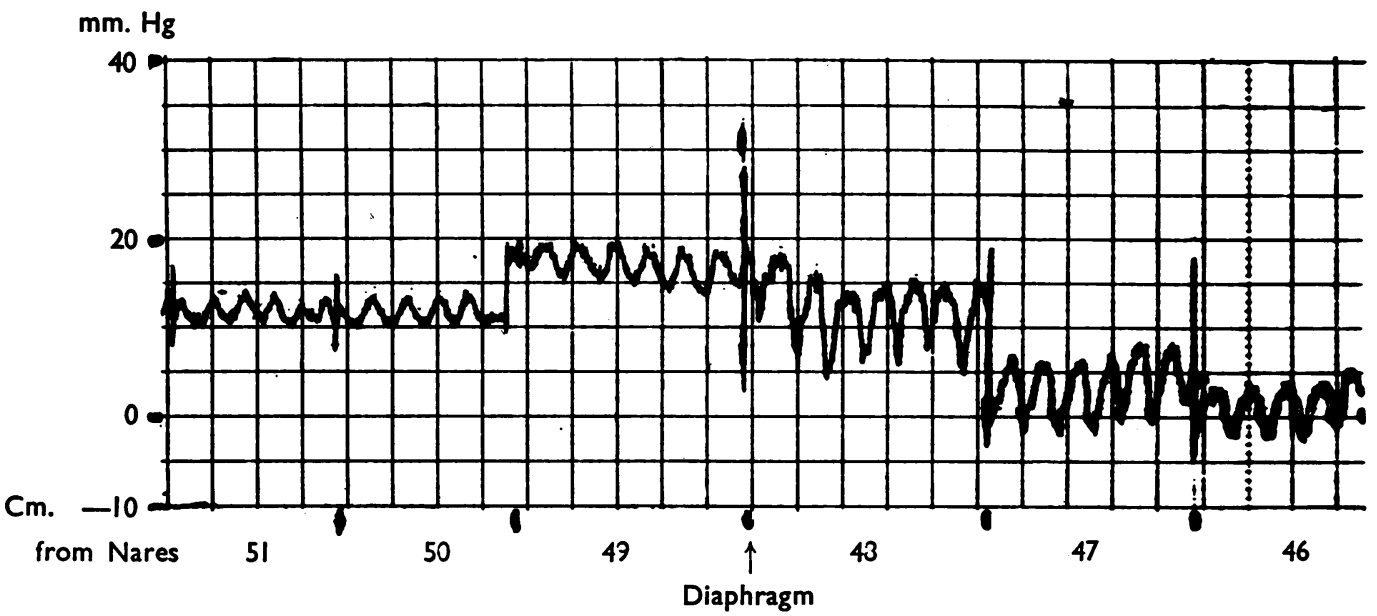

FIG. 1.-Pressure record obtained during withdrawal of an open-ended tube from the stomach to the oesophagus in $1 \mathrm{~cm}$. stages. Note the segment at the oesophago-gastric junction in which intraluminal pressure is raised.

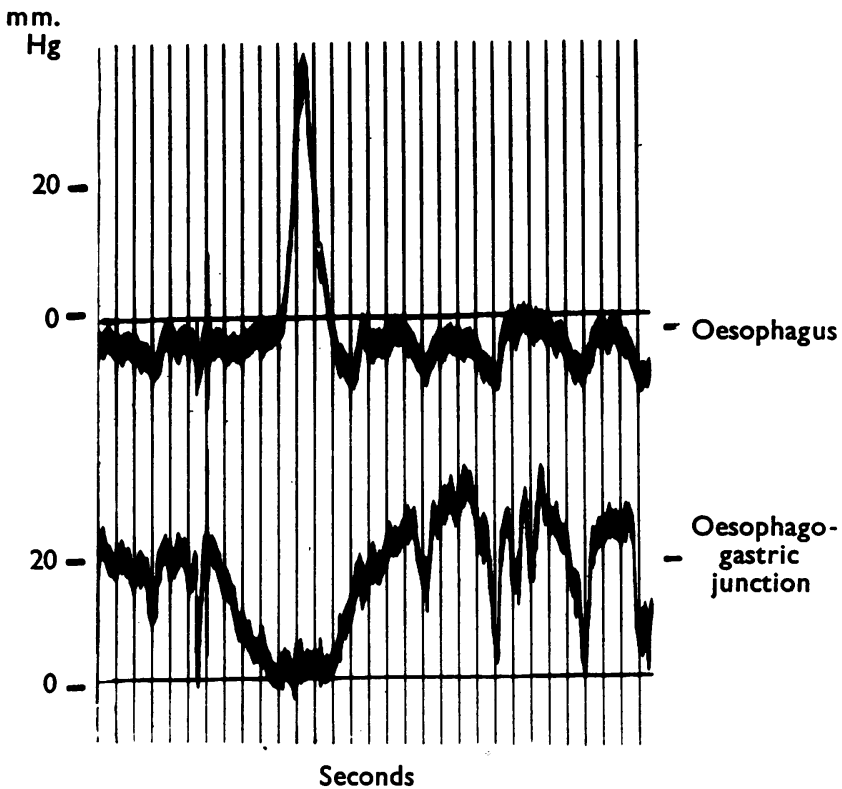

FIG. 2.-Simultaneous records obtained from two open-ended tubes during a dry swallow. Note the high resting pressure at the oesophago-gastric junction (lower record) and the temporary fall in pressure as the sphincter relaxes during the swallowing reflex.

subjects varied between 4 and $16 \mathrm{~mm}$. $\mathrm{Hg}$, with a mean of $8.4 \mathrm{~mm}$. $\mathrm{Hg}$.

Patients after Cardiomyotomy. - Records were obtained in 18 patients in each of whom a pressure recording tube was withdrawn from the stomach into the oesophagus six times.

The Oesophago-gastric Sphincter. - In two patients pressure measurements were made before and after cardiomyotomy. In each the estimated oesophago-gastric sphincteric tone was strikingly reduced after operation. The results are shown in Table $I$.

The records obtained from patient No. 1, a man of 25 giving a history of dysphagia of two years' duration, are shown in Fig. 3. Barium swallow revealed the typical appearances of achalasia of the cardia (Fig. 4). The preoperative record shows a prominent rise of pressure as the tip of the tube is withdrawn from the stomach into the sphincteric segment at $46 \mathrm{~cm}$. Intraluminal pressure in the oesophagus above the sphincter is higher than that found in normal subjeats. After operation the pressure elevation in the sphincteric segment is much less striking and intraoesophageal pressure has returned to normal levels.

In many patients studied after cardiomyotomy no increase in pressure was demonstrable at the oesophago-gastric junction, and in these it appeared that the function of the oesophago-gastric sphincter had been lost.

Fig. 5 is a record obtained from patient No. 14, a woman of 54 , who two years previously had undergone cardiomyotomy at another hospital. After the operation she developed a burning substernal pain with acid-tasting fluid rising into the mouth on lying flat in bed at night. Barium meal examination confirmed the presence of gastro-oesophageal reflux. The record shows no increase in pressure at the oesophago-gastric 

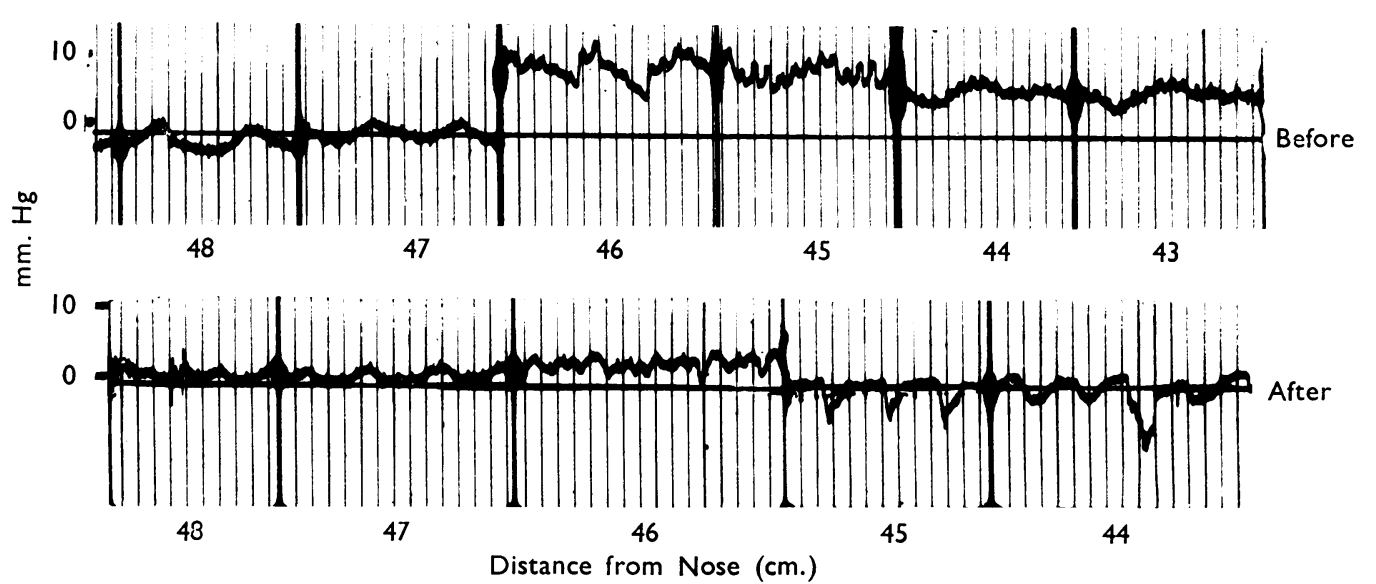

FIG. 3.-Records obtained from patient No. 1 during withdrawal of an open-ended tube from the stomach to the oesophagus before and afier cardiomyotomy. Note the prominent increase in pressure as the tube reaches the oesophago-gastric junction $(46 \mathrm{~cm}$.) before operation. After operation a much less striking rise of pressure is seen in this region.

junction, indicating that the oesophago-gastric sphincter had been completely disrupted by the operation.

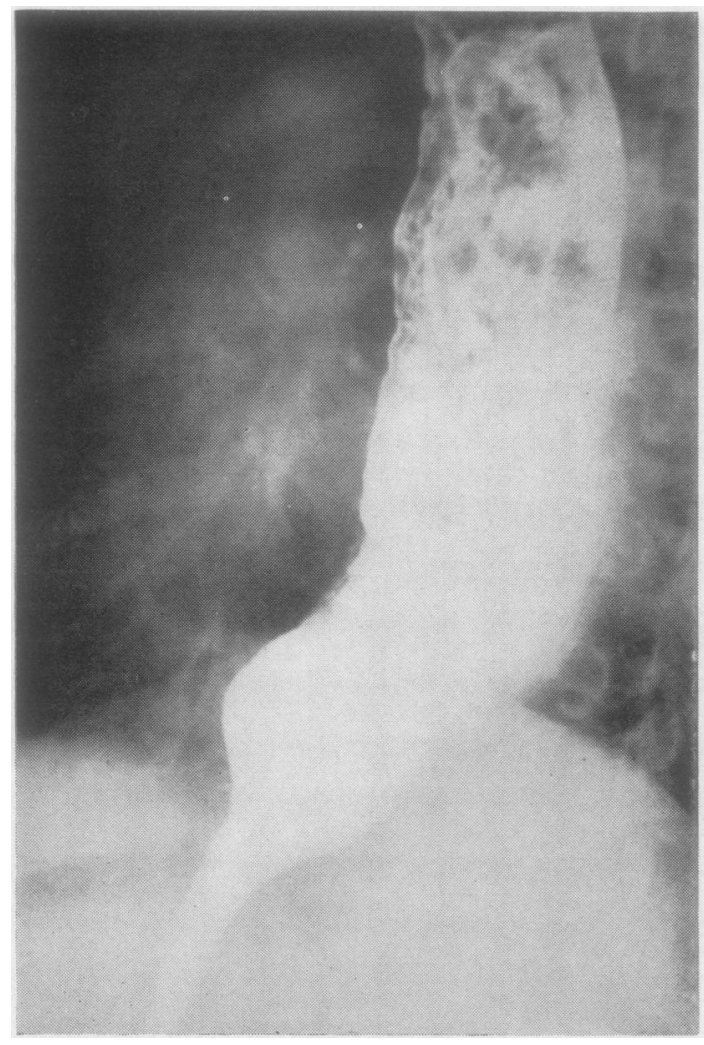

Fig. 4.-Radiograph (patient No. 1) before cardiomyotomy showing typical changes of achalasia of the cardia.
Using the pressure elevation in the sphincterice zone as a measure of the remaining tone of the oesophago-gastric sphincter, the values obtainece are shown in Table I. It will be seen that in many patients some sphincteric tone persisted after. operation and in some the values lay within the range found in normal subjects. When the patients were subdivided into those who showed symptoms of gastro-oesophageal reflux and those who did not (Fig. 6) it became obvious that the tone remaining in the oesophago-gastric sphincter was usually much greater in those who did not develop these symptoms. Four of the five patients with reflux showed no manometrically demonstrable sphincter and in the fifth a low value for sphincteric tone was obtained. Inn contrast, much higher values on the whole were obtained in the group with no reflux, and the difference between the two groups was statistically significant $(P=<0.01)$. This finding suggest $\frac{D}{5}$ that the residual tone of the sphincter after. cardiomyotomy is of importance in preventing tho development of reflux, and should some ton remain the patient is unlikely to suffer from reflux oesophagitis.

However, three patients (Nos. 8, 9, and 10) who had no symptoms of gastro-oesophageal reflux showed no manometric evidence of remaining tone in the sphincter. In these three patients the prevention of reflux must clearly have been 5 dependent upon some mechanism other than the oesophago-gastric sphincter.

The remaining tone in the oesophago-gastrie sphincter was within the range of normal in eack of the three patients (Nos. 11, 12, and 13) witl dysphagia persisting after operation. 
$\mathrm{mm} . \mathrm{Hg}$

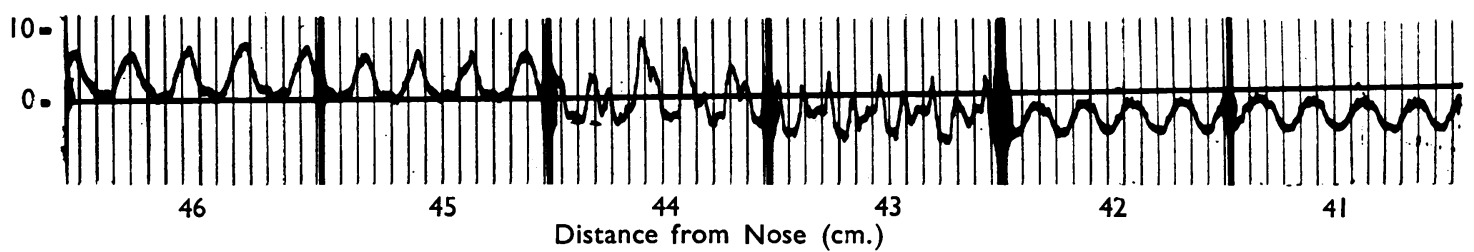

FIG. 5.-Record obtained fiom patient No. 14 during withdrawal of an open-ended tube from the stomach to the oesophagus showing no rise of pressure at the oesophago-gastric junction.

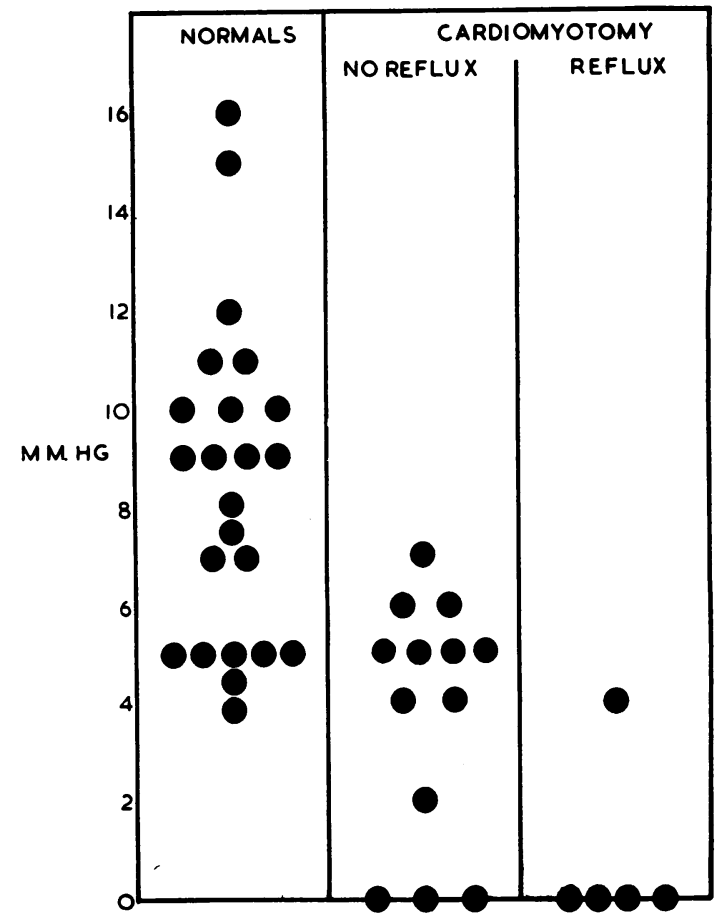

FIG. 6.-Estimated tone of the oesophago-gastric sphincter in relation to symptoms of reflux in 18 patients after cardiomyotomy. Sphincteric tone was estimated by subtracting expiratory pressure in the fundus of the stomach from that at the oesophago-gastric junction.

Oesophageal Motility.-Abnormality of the muscular activity of the oesophagus persisted in nearly all patients after cardiomyotomy. Both the patients studied before cardiomyotomy showed a high level of resting pressure in the oesophagus (Table I). After cardiomyotomy the resting pressure fell to the normal range in each of these patients, and in the majority of the other patients oesophageal resting pressure was within the range of normal. This fall in resting oesophageal pressure after cardiomyotomy probably represents a reduction in the tone of the oesophagus consequent upon the relief of the obstruction at the oesophago-gastric junction. It could not be explained simply as a reduction in the hydrostatic effect of the oesophageal contents resulting from more complete emptying, as neither of the two patients investigated before operation had a large residue at the time of the study and both were placed in the right lateral position to minimize hydrostatic effects.

Muscular activity after swallows of cold water was less prominent and less prolonged than that usually found in untreated achalasia. In five patients a single pressure wave of low amplitude occurred and in most of the remaining patients prolonged irregular muscular activity was seen.

\section{Discussion}

Manometric techniques provide a direct method of demonstrating the oesophago-gastric sphincter and assessing its functional state. These methods have been applied by Creamer, Olsen, and Code (1957) to the study of achalasia of the cardia, and have demonstrated that in this condition, although the tone of the oesophago-gastric sphincter is not increased, it shows complete failure of relaxation on swallowing.

These observations indicate that the oesophagogastric sphincter can frequently still be demonstrated after cardiomyotomy. This implies either that the sphincter is not completely disrupted at the time of operation or that some degree of regeneration occurs during the months or years following cardiomyotomy. Olsen, Schlegel, Creamer, and Ellis (1957), using manometric methods similar to those employed in this study, found that the subhiatal portion of the oesophago-gastric sphincter was preserved after cardiomyotomy and they believed that this is important in preventing reflux.

Their findings could not be confirmed in the present study; in seven patients no evidence of the oesophago-gastric sphincter either above or below the level of the diaphragm could be found, and in the remaining 11 , in whom the sphincter could still be demonstrated, this was frequently found to lie above the diaphragmatic hiatus. This discrepancy presumably results from a difference in the extent of the cardiomyotomy incision. In 
each of the patients in this series the incision was carried down on to the gastric wall for a distance of $2 \mathrm{~cm}$. or more to ensure that the whole of the sphincter was disrupted, whereas Olsen and others believe that the subhiatal portion should be preserved.

In this investigation, the height of the elevation of intraluminal pressure at the oesophago-gastric junction over that in the fundus of the stomach has been used as a measure of the residual tone of the oesophago-gastric sphincter. The values for residual sphincteric tone were significantly greater in those patients who did not develop symptoms of reflux after cardiomyotomy than in those who did, and it therefore seems likely that the persistence of some tone in the sphincter usually serves to prevent gastro-oesophageal reflux.

However, residual function of the oesophagogastric sphincter cannot be the only barrier to gastro-oesophageal reflux after cardiomyotomy, as three patients in this series had no manometric evidence of any remaining sphincteric tone and yet did not develop symptoms of reflux. The possibility that these patients in fact suffered from reflux but had insufficient acid and pepsin in the gastric juice to cause oesophagitis appeared unlikely, as the quinine indicator resin (" diagnex") test was done in one of them and showed free gastric acid. In these three patients at least, some factor other than the oesophagogastric sphincter was responsible for the prevention of the symptoms of gastro-oesophageal reflux.

It has previously been shown using similar techniques that, in the presence of hiatus hernia with displacement of the cardia into the thorax, the tone of the oesophago-gastric sphincter is of cardinal importance in preventing reflux (Atkinson and others, 1957b). It would appear that when the cardia is displaced into the chest this sphincter forms the only important barrier to reflux, and that, if it loses its tone, reflux will almost certainly occur. Hiatus hernia occasionally develops after cardiomyotomy and nearly always results in reflux, as the barrier formed by the sphincter has already been removed.

The factor responsible for the competence of the cardia after complete destruction of the oesophago-gastric sphincter appears only to be operative so long as the stomach and oesophagus retain their normal anatomical positions. The nature of this factor has been a subject of controversy for many years. External compres- sion of the oesophago-gastric junction by the $\overrightarrow{\bar{S}}$ diaphragm (Jackson, 1922 ; Allison, 1951), or a mucosal rosette pulled together by the contraction of the muscularis mucosa (Dornhorst, Harrison, and Pierce, 1954; Botha, 1958), have been suggested as possible mechanisms. However, each of these would cause direct compression of a pressure recording at the oesophago-gastric junction and would therefore be expected to produce an increase in intraluminal pressure in this region. No such increase could be recorded in three patients who showed no clinical evidence of gastro-oesophageal reflux. Therefore, it seems unlikely that these mechanisms were responsible for the continued competence of the cardia.

The answer to this problem may lie in the well- established observation that operations for $\subseteq$ achalasia, which result in gross anatomical disturbance of the oesophago-gastric junction, $\vec{\varphi}$ such as cardioplasty or resection of the cardia on with oesophago-gastric anastomosis, are followed by a much higher incidence of gastro-oesophageal reflux than is cardiomyotomy. This provides suggestive evidence to support the view that the normal oblique angle of entry of the oesophagus to the stomach is of importance in maintaining the competence of the cardia by forming a $\overrightarrow{\overrightarrow{0}}$ mechanical valve arrangement (von Gubaroff, 3 1886 ; Collis, Kelly, and Wiley, 1954), as after the earlier operations this angle was lost or distorted. The manometric findings after cardiomyotomy would be compatible with the view that in preventing reflux the action of the oesophago-gastric sphincter is supplemented by such a mechanical valve arrangement.

\section{SUMMARY}

The functional state of the oesophago-gastric sphincter has been assessed manometrically in 18 음 patients, who had undergone cardiomyotomy one month to nine years previously. Eleven showed of some evidence of remaining function, whilst in $N$ seven the sphincter was no longer demonstrable. N

In those patients who suffered from gastro- $\frac{\omega}{\sigma}$ oesophageal reflux after the operation, the impairment of function of the oesophago-gastric sphincter was greater than in those without reflux, suggesting that residual tone in the sphincter is of importance in preventing reflux after cardiomyotomy.

However, no sphincter could be demonstrated $\frac{\rho}{\mathbb{D}}$ in three patients who did not develop symptoms $\frac{\varrho}{\sigma}$ of reflux. Some additional factor must have been responsible for the competence of the cardia in 
these patients and the manometric findings would be most compatible with a mechanical valve arrangement.

I wish to thank Mr. H. Revell for his unstinted technical help and the Medical Research Council for a grant for apparatus. I am grateful to Mr. Geoffrey Wooler and to Mr. J. A. Aylwin for allowing me to study patients under their care, and to Dr. W. Whitaker for access to the technical facilities of the cardiac department.

\section{REFERENCES}

Acheson, E. D., and Hadley, G. D. (1958). Brit. med. J., 1, 549. Allison, P. R. (i951). Surg. Gynec. Obstet., 92, 419.

Atkinson, M., Edwards, D. A. W., Honour, A. J., and Rowlands,

E. N. (1957a). Lancet, 2, $918 ., 2,1138$.
Barrett, N. R., and Franklin, R. H. (1949). Brit. J. Surg., 37, 194. Botha, G. S. M. (1958). Ibid., 45, 569.

- Astley, R., and Carré, I. J. (1957). Lancet, 1, 659.

Brewer, M.S., Barnes, W. A., and Redo, S. F. (1956). Ann. Surg. 144,823 .

Collis, J. L., Kelly, T. D., and Wiley, A. M. (1954). Thorax, 9, 175 Creamer, B., Olsen, A. M., and Code, C. F. (1957). Gastroenterology, $33,293$.

Dornhorst, A. C., Harrison, K., and Pierce, J. W. (1954). Lancet, 1,695 .

Fyke, F. E., Jr., Code, C. F., and Schlegel, J. F. (1956). Gastroenterologia (Basel), 86, 135 .

Gammie, W. F. P., Jennings, D., and Richardson, J. E. (1958). Lancet, $2,917$.

Gertz, T. C. (1952). Acta chir. scand . 103, 459

Gubaroff, A von (1886). Arch. Anat. Physiol. (Anat. Abt.) (Lpz.), p. 395

Hawthorne, H. R., Frobese, A. S., and Nemir, P., Jr. (1956). Ann. Surg., 144,653 .

Jackson, C. (1922). Laryngoscope (St. Louis), 32, 139

Olsen, A. M., Schlegel, J. F., Creamer, B., and Ellis, F. H. (1957) $J$, thorac Surg 34,615 .

Ripley, H. R., Olsen, A. M., and Kirklin, J. W. (1952). Surgery, 32, 1 . 